\title{
EXTENDING COHERENT ANALYTIC SHEAVES THROUGH SUBVARIETIES
}

\author{
BY YUM-TONG SIU ${ }^{1}$ \\ Communicated by Arthur Mattuck, July 29, 1968
}

We announce the following

THEOREM I. Suppose $V$ is a subvariety of dimension $\leqq n$ in a (not necessarily reduced) complex space $X$ and $\mathcal{F}$ is a coherent analytic sheaf on $X-V$ with codh $\Im \geqq n+3$. Let $\theta: X-V \rightarrow X$ be the inclusion map. Then $\theta_{0}(\mathcal{F})$ is a coherent analytic sheaf on $X$ extending $\mathcal{F}$ (where $\theta_{q}(\mathcal{F})$ is the qth direct image of $\mathcal{F}$ under $\theta$ ).

The case $n=0$ was proved in [7]. The case where $X$ is a manifold of dimension $n+3$ was proved in [5].

We give here only a very brief outline of the proof together with some related results and application. Details will appear elsewhere.

Suppose $\mathcal{F}$ is an analytic sheaf on a complex space $X$ and $n$ is a nonnegative integer. We denote by $\mathcal{F}^{[n]}$ the analytic sheaf on $X$ defined by the following presheaf: if $U \subset W$ are open subsets of $X$, then $\mathcal{F}^{[n]}(U)=$ the direct limit of $\{\Gamma(U-A, \mathfrak{F}) \mid A \in \mathfrak{A}\}$, where $\mathfrak{A}$ is the set of all subvarieties of dimension $\leqq n$ in $U$ directed by inclusion, and $\mathfrak{F}^{[n]}(W) \rightarrow \mathfrak{F}^{[n]}(U)$ is induced by restriction maps. There is a canonical sheaf-homomorphism from $\mathscr{F}$ to $\mathcal{F}^{[n]}$. We denote by $O_{[n] \mathscr{F}}$ the analytic subsheaf of $\mathcal{F}$ defined as follows: for $x \in X, s \in\left(O_{[n] F}\right)_{x}$ if and only if there exist an open neighborhood $U$ of $x$ in $X$, a subvariety $A$ in $U$ of dimension $\leqq n$, and $t \in \Gamma(U, \Im)$ such that $t_{x}=s$ and $t_{y}=0$ for $y \in U-A$.

Proposition 1 [6]. Suppose $\mathcal{F}$ is a coherent analytic sheaf on a complex space $X$ and $n$ is a nonnegative integer.

(a) If $O_{[n+1] \mathcal{F}}=0$, then $\mathcal{F}^{[n]}$ is coherent and the subvariety where $\mathcal{F}^{[n]}$ is not isomorphic to $\mathcal{F}$ canonically is of dimension $\leqq n$.

(b) If $\mathcal{F}$ is canonically isomorphic to $\mathcal{F}^{[n]}$, then $O_{[n+1] \mathcal{F}}=0$.

The following can be proved from Proposition 1 and by induction on $n$.

Proposition 2. Suppose $\mathfrak{F}$ is a coherent analytic sheaf on a complex space $X$ and $n$ is a nonnegative integer such that $F$ is canonically iso-

1 Supported partially by NSF Grant GP-7265. 
morphic to $\mathcal{F}^{[n]}$. Then for $-1 \leqq k<n$ the subvariety $\left\{x \in X \mid \operatorname{codh} \mathcal{F}_{x}\right.$ $\leqq k+2\}$ has dimension $\leqq k$.

Let $z_{1}, \cdots, z_{N}$ and $t_{1}, \cdots, t_{n}$ be respectively coordinates of $C^{N}$ and $\boldsymbol{C}^{n}$. Let $\mathcal{O}$ and $\mathcal{\theta}$ be respectively the structure-sheaves of $\boldsymbol{C}^{n}$ and $\boldsymbol{C}^{N} \times \boldsymbol{C}^{n}$. For $0 \leqq a<b$ and $\rho>0$, let

$$
\begin{aligned}
G(b) & =\left\{z \in C^{N} \mid \max \left(\left|z_{1}\right|, \cdots,\left|z_{N}\right|\right)<b\right\}, \\
G(a, b) & =\left\{z \in G(b)|a<| z_{i} \mid \text { for some } 1 \leqq i \leqq N\right\},
\end{aligned}
$$

and $K(\rho)=\left\{t \in C^{n} \mid \max \left(\left|t_{1}\right|, \cdots,\left|t_{n}\right|\right)<\rho\right\} . K=K(1)$. Let $\pi: G(a, b) \times K \rightarrow K$ be the projection.

Proposition 3. Suppose $0<\tilde{a}<a<b<\tilde{b}$ and $F$ is a coherent analytic sheaf on $G(\tilde{a}, \tilde{b}) \times K$ such that $\operatorname{codh} F \geqq n+3$ and $t_{n}$ is not a zero-divisor for $\mathcal{F}_{x}$ for $x \in G(\tilde{a}, \tilde{b}) \times 0$. Suppose $\mathcal{F} / t_{n} \mathfrak{F}$ can be extended to a coherent analytic sheaf $\mathcal{g}$ on $G(\tilde{b}) \times K$ such that $\operatorname{codh} g \geqq n+1$ and $t_{1}, \cdots, t_{n-1}$ is a $\mathrm{g}_{x}$-sequence for $x \in G(\tilde{b}) \times 0$. Then $\left(\pi_{1}(\Im)\right)_{0}$ is finitely generated over $\mathcal{O}_{0}$.

The proof of Proposition 3 is rather complicated where modifications of techniques of [1] and [2] are used.

Proposition 4. Suppose $a, b, \tilde{a}, \tilde{b}$, and $\mathfrak{F}$ are as in Proposition 3. If $z_{j}-z_{j}(x)$ is not a zero-divisor for $\mathfrak{F}_{x}$ for $x \in G(\tilde{a}, \tilde{b}) \times K$ and $1 \leqq j \leqq N$. Then for some $a<c<d<b$ and $0<\rho<1 \Gamma(G(c, d) \times K(\rho), \mathfrak{F})$ generates F on $G(c, d) \times K(\rho)$.

Proof (Sketch). Consider

$\left({ }^{*}\right)_{k}$ For some $a<c<d<b$ and $0<\rho<1$ there exists a subvariety $Z$ in $G(c, d) \times K(\rho)$ such that $\Gamma(G(c, d) \times K(\rho), \mathscr{F})$ generates $\mathfrak{F}$ on $G(c, d) \times K(\rho)-Z$ and $\operatorname{dim} Z \cap G(c, d) \times 0 \leqq k$.

The Proposition follows by proving $\left({ }^{*}\right)_{b}$ by backward induction on $k$ for $0 \leqq k \leqq N$. For the induction process we need only prove the following.

( $\dagger$ ) If $Z$ is a positive-dimensional subvariety of $G(a, b) \times 0$, then for some $x \in Z$ and some $0<\rho<1 \Gamma\left(G(a, b) \times K(\rho)\right.$, F) generates $\mathfrak{F}_{x}$.

To prove ( $\dagger$ ), choose $1 \leqq j \leqq N$ and $\left\{x_{m}\right\}_{m=1}^{\infty} \subset Z$ such that $\left|z_{j}\left(x_{m}\right)\right|>a$ and $\left|z_{j}\left(x_{m}\right)\right| \rightarrow b$. Let $V_{m}=\left\{x \in G(a, b) \times K \mid z_{j}(x)=z_{j}\left(x_{m}\right)\right\} \quad$ and $V=\bigcup_{m=1}^{\infty} V_{m}$. Let $f$ be a holomorphic function on $G(b) \times K$ generating the ideal-sheaf of $V$. The short exact sequence $0 \rightarrow \mathfrak{F} \stackrel{\oplus}{\rightarrow} \mathcal{F} \rightarrow \mathfrak{F} / f \mathfrak{F} \rightarrow 0$ (where $\phi$ is defined by multiplication by $f$ ) gives rise to the exact sequence 


$$
\left(\pi_{0}(\mathcal{F})\right)_{0} \stackrel{\alpha}{\rightarrow}\left(\pi_{0}(\mathcal{F} / f \mathcal{F})\right)_{0} \stackrel{\beta}{\rightarrow}\left(\pi_{1}(\mathcal{F})\right)_{0} .
$$

Let $\gamma: \tilde{\mathcal{O}}^{p} \rightarrow \mathcal{F}$ be a sheaf-epimorphism on $\left\{x \in G(b) \times K\left(\frac{1}{2}\right)|a<| z_{j}(x) \mid\right\}$. $\boldsymbol{\gamma}$ induces $\boldsymbol{\gamma}^{\prime}: \tilde{\mathcal{O}}^{p} / f^{\sim} p \rightarrow \mathcal{F} / f \mathcal{F}$. Let $s_{m}^{(i)} \in\left(\pi_{0}(\mathcal{F} / f \mathcal{F})\right)_{0}$ be induced under $\gamma^{\prime}$ by the $p$-tuple of holomorphic functions on $V$ which is $(0, \cdots, 0,1,0, \cdots, 0)$ on $V_{m}$ with 1 in the $i$ th place and is zero otherwise. By considering the direct sum of $p$ copies of (\#) and using Proposition 3 we obtain $a_{1}, \cdots, a_{m-1} \in \mathcal{O}_{0}$ for some $m$ such that for all $1 \leqq i \leqq p \beta\left(s_{m}^{(i)}-\sum_{q=1}^{m-1} a_{q} s_{q}^{(i)}\right)=0$. For some $t_{m}^{(1)}, \cdots, t_{m}^{(p)}$ $\in\left(\pi_{0}(\mathcal{F})\right)_{0}, \alpha\left(t_{m}^{(i)}\right)=s_{m}^{(i)}$. $\mathcal{F}_{x_{m}}$ is generated by sections of $\mathcal{F}$ inducing $t_{m}^{(1)}, \cdots, t_{m}^{(p)}$.

Q.E.D.

Proposition 5. Suppose $D$ is a domain in $C^{n}, 0 \leqq a<b$, and $\mathcal{F}$ is a coherent analytic sheaf on $G(b) \times D$. If $F^{[n-1]} \approx \mathfrak{F}$, then the restriction map $\phi: \Gamma(G(b) \times D, F) \rightarrow \Gamma(G(a, b) \times D, F)$ is injective. If $\mathcal{F}^{[n]} \approx \mathcal{F}$, then $\phi$ is surjective.

Proof (Sketch). The injectivity of $\phi$ follows from Proposition 1 (b). For the surjectivity of $\phi$ consider first the special case codh $F$ $\geqq n+2$. For the general case use Proposition 2 and induction on $\boldsymbol{n}$.

Q.E.D.

Proposition 6. Suppose $D$ is a domain in $C^{n}, 0 \leqq a<a^{\prime}<b$, and $\mathfrak{F}$ is a coherent analytic sheaf on $G(a, b) \times D$ with $\mathfrak{F}^{[n+1]} \approx \mathfrak{F}$. Then the restriction map $\Gamma(G(a, b) \times D, F) \rightarrow \Gamma\left(G\left(a^{\prime}, b\right) \times D, \mathfrak{F}\right)$ is bijectrve.

Proof (Sketch). Use Proposition 5 and consider the restriction maps $\Gamma\left(\left(G(a, b) \cap U_{i}\right) \times D, F\right) \rightarrow \Gamma\left(\left(G\left(a^{\prime}, b\right) \cap U_{i}\right) \times D, \mathcal{F}\right)$ and $\Gamma\left(\left(G(a, b) \cap U_{i} \cap U_{j}\right) \times D, F\right) \rightarrow \Gamma\left(\left(G\left(a^{\prime}, b\right) \cap U_{i} \cap U_{j}\right) \times D, \mathcal{F}\right)$, where $U_{i}=\left\{x \in C^{N}|| z_{i}(x) \mid>a\right\}$.

By using Propositions 1, 2, 4, 5, and 6 and by induction on $n$, we can obtain

Theorem II. Suppose $D$ is a domain in $\mathrm{C}^{N}, 0 \leqq a<b$, and $\mathcal{F}$ is a coherent analytic sheaf on $G(a, b) \times D$ with $\mathcal{F}^{[n+1]} \approx \mathcal{F}$.

(a) There exists a coherent analytic sheaf $\mathcal{F}^{\prime}$ on $G(b) \times D$ which extends $\mathcal{F}$ and satisfies $\left(\mathfrak{F}^{\prime}\right)^{[n+1]} \approx \mathfrak{F}^{\prime}$.

(b) If $\mathcal{F}^{\prime}$ and $\mathcal{F}^{\prime \prime}$ are two coherent analytic sheaves on $G(b) \times D$ both extending $\mathfrak{F}$ such that $\left(\mathfrak{F}^{\prime}\right)^{[n+1]} \approx \mathfrak{F}^{\prime}$ and $\left(\mathcal{F}^{\prime \prime}\right)^{[n+1]} \approx \mathfrak{F}^{\prime \prime}$, then there exists a unique sheaf-isomorphism from $\mathfrak{F}^{\prime}$ to $\mathfrak{F}^{\prime \prime}$ which is equal to the identity map of $\mathcal{F}$ on $G(a, b) \times D$.

As a corollary of Theorem II we have

Theorem III. Suppose $V$ is a subvariety of dimension $\leqq n$ in a com- 
plex space $X$ and $\mathcal{F}$ is a coherent analytic sheaf on $X-V$. If $\mathfrak{F}^{[n+1]} \approx \mathcal{F}$, then $\theta_{0}(\mathcal{F})$ is a coherent analytic sheaf on $X$ extending $\mathcal{F}$, where $\theta: X-V$ $\rightarrow X$ is the inclusion map.

Theorem I follows from Theorem III and Korollar zu Satz III of [3].

Theorem III answers in the affirmative the following question posed by Serre [4, p. 372]: Suppose $V$ is a subvariety of codimension $\geqq 3$ in a normal reduced complex space $X$. If $\mathcal{F}$ is a reflexive coherent analytic sheaf on $X-V$, is $\theta_{0}(\mathcal{F})$ coherent (where $\theta: X-V \rightarrow X$ is the inclusion map)?

\section{REFERENCES}

1. A. Andreotti and $\mathrm{H}$. Grauert, Théorèmes de finitude pour la cohomologie des espaces complexes, Bull. Soc. Math. France 90 (1962), 193-259.

2. H. Grauert, Ein Theorem der analytischen Garbentheorie und die Modulräume komplexer Strukturen, Inst. Hautes Etudes Sci. Publ. Math. No. 5 (1960).

3. G. Scheja, Riemannsche Hebbarkeitssätze für Cohomologieklassen, Math. Ann. 144 (1961), 345-360.

4. J -P. Serre, Prolongement de faisceaux analytiques cohérents, Ann. Inst. Fourier (Grenoble) 16 (1966), 363-374.

5. Y.-T. Siu, Extension of locally free analytic sheaves, Math. Ann. (to appear).

6. - Absolute gap-sheaves and extensions of coherent analytic sheaves (to appear).

7. G. Trautmann, Ein Kontinuilätssatz für die Fortsetzung kohärenter analytischer Garben, Arch. Math. (Basel) 18 (1967), 188-196.

University of Notre Dame, Notre Dame, Indiana 46556 